Here, we took a systems biology approach to characterize phosphatases regulating mitosis. We performed a genome-wide RNAi screen targeting all human phosphatases.

We discovered several novel mitotic phosphatases, including CDKN3, and we have shown that CDKN3 inactivates cyclindependent kinases at the exit from mitosis by dephosphorylating Thr-161 of CDC2. We demonstrated that CDKN3 and CDC2 colocalize on centrosomes during mitosis and that loss of CDKN3 disrupts centrosome maintenance. We analyzed a phosphoproteome landscape of

CDKN3-deficient cells to reveal that CDKN3 knockdown leads to abnormal phosphorylation of multiple downstream cell cycle proteins, including CK $\beta$. We have shown that CK $\beta$ phosphorylated at Ser-209 regulates the spindle checkpoint and localizes to centrosomes during mitosis. We confirmed that CDKN3 is required for mitosis in primary human brain stem cells, and we found that CDKN3 is lost in the glioblastoma multiforme brain tumors.

In summary, we have discovered a novel CDKN3/CDC2/CK $\beta$ tumor suppressor signaling axis. Our findings have diagnostic and therapeutic importance in cancer. Our discoveries enhance our comprehension of the cross-talk between mitotic phosphorylation cascades that maintain genomic stability. This signaling axis is a viable anti-cancer target in glioblastoma multiforme and other malignancies. Pre-clinical and clinical trials of small molecules targeting this signaling axis may lead to discoveries of novel anti-cancer chemotherapy strategies.

\section{CIRCULATING PRO-ENDOTHELIN-1 (CT-PROET-1) AND PULMONARY MORBIDITY IN NEWBORN INFANTS}

doi:10.1136/archdischild-2012-302724.0200

1,2S Wellmann, ${ }^{2} \mathrm{~J}$ Benzing, ${ }^{1} 0$ Stabile, ${ }^{2} \mathrm{G}$ Szinnai, ${ }^{3} \mathrm{NG}$ Morgenthaler, ${ }^{2} \mathrm{SM}$ Schulzke, ${ }^{3} \mathrm{C}$ Bührer. 'University Hospital Zurich, Zurich; 'University Children's Hospital Basel, Basel, Switzerland; ${ }^{3}$ Charité University Medical Centre, Berlin, Germany

Background and aims Plasma concentrations of endothelin-1 (ET-1), a potent pulmonary vasoconstrictor with a short serum half-life, can be estimated by measuring its stable by-product, C-terminal pro-Endothelin-1 (CT-proET-1). To investigate the association between CT-proET-1, gestational age, and pulmonary morbidity in newborn infants.

Methods A prospective cross-sectional study at two tertiary university hospitals was conducted. CT-proET-1 concentrations (pmol/L) were determined in plasma samples of 293 newborn infants (gestational age 24-41 weeks) at birth and on day 3 of life with BRAHMS KRYPTOR automated immunoflorescent assay.

Results At birth, CT-proET-1 concentrations were unrelated to birth weight and gestational age. Venous umbilical cord CT-proET-1 concentrations were consistently higher than matched arterial ones (M 148 vs. $134 \mathrm{pmol} / \mathrm{L}, \mathrm{p}<0.001$ ), but both values were closely related $\left(R_{s}=0.745, p<0.001\right)$. There was large postnatal increase of CT-proET-1 in infants with pulmonary morbidity. Days of mechanical ventilation, continuous positive airway pressure (CPAP), and oxygen supplementation correlated each with CT-proET-1 level on day $3\left(R_{s} 0.566,0.658\right.$, and 0.819 , respectively, for all $\left.p<0.001\right)$.

Conclusions In term and preterm newborn infants, pulmonary compromise is associated with increased concentrations of circulating CT-proET-1.

\section{EFFICACY OF MONOTHERAPY WITH INHALED NITRIC OXIDE VERSUS COMBINATION WITH ORAL SILDENAFIL IN PERSISTENT PULMONARY HYPERTENSION OF THE NEWBORN}

SS Oguz, HG Kanmaz, E Dizdar Alyamac, FN Sari, 0 Erdeve, U Dilmen. Zekai Tahir Burak Maternity Teaching Hospital Neonatology Unit, Ankara, Turkey

Objective To evaluate the efficacy of combined therapy, sildenafil and inhaled nitric oxide versus monotherapy with inhaled nitric oxide in Pulmonary Hypertension of the Newborn.

Study Design A retrospective study.

Patients and Methods Newborn infants (gestational age greater than 34 weeks) who were presented with pulmonary hypertension from December 2008 to December 2010 were evaluated. Group I $(n=14)$ received monotherapy with iNO and Group II $(n=9)$ received combination therapy with iNO and oral sildenafil. Main outcome was to compare the duration of iNO therapy between groups.

Results Demographic characteristics were similar between the groups. As compared with the groups, combined therapy group had higher mean (SD) age of NICU admission (5.1 $\pm 8.2 \mathrm{~h}$ vs. $21.3 \pm 36, \mathrm{~h}$, $\mathrm{p}=0.01)$. Combination therapy was associated with early weaning of iNO (4.8 \pm 1.5 vs. $13.5 \pm 7.6$ hours). The result showed that there is a reduced need for iNO therapy compared to monotherapy $(75 \pm 44.6$ vs. $112 \pm 95.2)$, however, the difference was insignificant $(p=0.36)$. The incidence of mortality and outcomes were statistically insignificant between the groups ( $p>0.05)$.

Conclusions Combination therapy is significantly more effective in weaning of iNO and reduces the need for iNO therapy that is relatively expensive.

\section{SURFACTANT AND INHALED NITRIC OXIDE FOR SEVERE RESPIRATORY DISTRESS COMPLICATED WITH PERSISTENT PULMONARY HYPERTENSION AMONG INFANTS BELOW 32 WEEKS GA}

doi:10.1136/archdischild-2012-302724.0202

'A Adamczak, ${ }^{2} \mathrm{~S}$ Gajos, ${ }^{2} \mathrm{~A}$ Mejsak, ${ }^{3} \mathrm{~T} A$ Merritt, 'J Gadzinowski, 'J Mazela. ${ }^{1}$ Neonatology; ${ }^{2}$ Neonatal Research Club, Poznan University of Medical Sciences, Poznan, Poland; '3Pediatrics, Loma Linda Medical Center, Loma Linda, CA, USA

Introduction RDS can be complicated by PPHN due to intrauterine or postnatal prolonged hypoxia regardless of surfactant replacement therapy (SRT). Use of iNO therapy is not approved for premature infants $<34$ weeks, nevertheless in most severe cases of PPHN use of iNO has been reported.

Aim The aim of this study was to analyze outcomes of infants with severe RDS treated with SRT and iNO in comparison to those treated with SRT only.

Methods Medical records from January 2008 till December 2010 from a level III NICU were analyzed. Only infants $<32$ weeks ga and treated with SRT for RDS were included in the study divided in two groups: treated with SRT+iNO (PPHN based on $\mathrm{SaO}_{2}$ differences and echocardiography) and SRT only. Data were analyzed according to maternal history: intrauterine infection, PROM, and clinical outcomes: pneumonia, NEC, ROP, BPD, IVH.

Results 309 premature infants $<32$ weeks gestation were treated with SRT, with 54(17\%) treated with iNO due to PPHN. There were significant higher ratios of intrauterine infection and early pneumonia in SRT+iNO in 2008 and 2009. The neonatal outcomes showed consistent higher incidence of ROP and PVL for the SRT+iNO group in these same years. The BPD rate remained unchanged at $20 \%$ and mortality ranged from 18 to $48 \%$.

Conclusions Severe PPHN was likely caused by ineffective SRT due to presence of inflammation and possible surfactant inactivation. Inhaled NO improved oxygenation and decreased signs of PPHN but did not influence rate of BPD.

\section{THE AVON LONGITUDINAL STUDY OF PAREANTS AND CHILDREN (ALSPAC) - WHAT DID WE LEARN?}

doi:10.1136/archdischild-2012-302724.0203 